\title{
BIOPELÍCULAS FOTOACTIVAS: MATERIAL DE EMPAQUE EN ALIMENTOS SENSIBLES A LA OXIDACIÓN
}

\section{PHOTOACTIVE BIOFILMS: PACKAGING MATERIALS IN OXIDATION SENSITIVE FOODS}

\author{
Lina Johanna Niño Otálora ${ }^{1}$, Angélica María García Torres ${ }^{2 *}$, Oscar Julio Medina Vargas ${ }^{3}$, Carlos Iván Rojas Morales ${ }^{4}$
}

\begin{abstract}
${ }^{1}$ Química. Universidad Pedagógica y Tecnológica de Colombia, Grupo de Investigación en Química y Tecnología de Alimentos, Avenida Central del Norte 39-115, Tunja-Boyacá, Colombia, e-mail: linajohanna.nino@uptc.edu.co, (iDhttps://orcid. org/0000-0001-5335-6172; ²uímica, PhD. Docente. Universidad Pedagógica y Tecnológica de Colombia, Escuela de Ciencias Químicas, Grupo de Investigación en Química y Tecnología de Alimentos, Avenida Central del Norte 39-115, Tunja-Boyacá, Colombia, email: angela.garciatorres@uptc.edu.co, (iDhttps://orcid.org/0000-0003-4014-7931; ${ }^{3}$ Licenciado en Química y Biología, PhD en Ciencia y Tecnología de Alimentos, Docente. Universidad Pedagógica y Tecnológica de Colombia, Escuela de Ciencias Químicas, Grupo de Investigación en Química y Tecnología de Alimentos, Avenida Central del Norte 39-115, Tun-

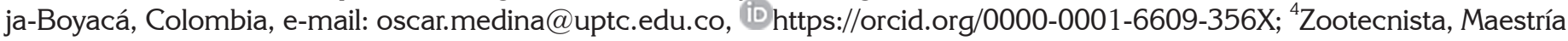
en Ciencias Biológicas. Universidad Pedagógica y Tecnológica de Colombia, Grupo de Investigación en Química y Tecnología de Alimentos, Avenida Central del Norte 39-115, Tunja-Boyacá, Colombia, e mail: carlos.rojas09@uptc.edu.co, (iDhttps:// orcid.org/0000-0002-3330-7469; *autor para correspondencia: angela.garciatorres@uptc.edu.co
\end{abstract}

\author{
Rev. U.D.C.A Act. \& Div. Cient. 21(2):457-466, Julio-Diciembre, 2018 \\ https://doi.org/10.31910/rudca.v21.n2.2018.1080
}

Artículo de acceso abierto publicado por Revista U.D.C.A Actualidad \& Divulgación Científica bajo una licencia Creative Commons CC BY-NC 4.0

\section{RESUMEN}

La contaminación, a causa de residuos plásticos, es uno de los principales problemas ambientales, emergentes del siglo XX. Particularmente, el uso de envases plásticos para alimentos y bebidas ocupa el $50 \%$ de su producción total. La producción de empaques bioplásticos, a partir de materias primas renovables, surge como alternativa de bajo impacto ambiental en la industria de alimentos, sensibles a la oxidación, conservando su calidad organoléptica y nutricional. Esta investigación tuvo como objetivo la elaboración de biopelículas, a partir de almidón residual esterificado de papa, anclado a sustancias fotoactivas, con diferentes rangos de absorción electromagnética: betalaína, rutina y riboflavina, capaces de disminuir los procesos oxidativos inducidos por la luz, en muestras de carne bovina. Se evaluaron las propiedades fisicoquímicas, mecánicas y de eficacia fotoactiva. Los resultados mostraron que el anclaje de las sustancias fotoactivas al almidón esterificado, optimizó los valores de solubilidad en agua, transparencia, estabilidad ácido-base y resistencia a la ruptura de las biopelículas. La mayor protección fotoactiva, se obtuvo con la biopelícula de rutina, reduciendo en un $66,6 \%$ y $57,3 \%$ la degradación oxidativa de proteínas y lípidos de la carne. Se concluye, que la esterificación del almidón residual de papa y su posterior anclaje de sustancias fotoactivas, le confiere un uso potencial en la producción de envases biodegradables para alimentos.

Palabras clave: biopelículas, empaque de alimentos, sustancias fotoactivas, almidón, degradación oxidativa.

\section{ABSTRACT}

Pollution caused by plastic waste is one of the main emerging environmental problems of the 20th century. Particularly the use of plastic containers for food and beverages that occupies almost $50 \%$ of its total production. The elaboration of bioplastics from renewable raw materials emerges as an alternative of low environmental impact in the food industry sensitive to oxidation, conserving its organoleptic and nutritional quality. The aim of this research was to prepare biofilms from residual esterified potato starch, anchored to photoactive substances with different ranges of electromagnetic absorption: Betalaine, Rutin and Riboflavin, able to reduce oxidative processes induced by light in bovine meat samples. The physicochemical, mechanical 
and photoactive efficiency properties were evaluated. The results showed that the anchoring of the photoactive substances to the esterified starch optimizes the values of water solubility, transparency, acid-base stability and resistance to rupture of the biofilms. The greater photoactive protection was obtained with the biofilm of Rutin, reducing in $66.6 \%$ and $57.3 \%$ the oxidative degradation of proteins and lipids respectively. It is concluded that the esterification of residual potato starch and its subsequent anchoring of photoactive substances confers a potential use in the production of biodegradable food packaging.

Keywords: biofilms, food packaging, photoactive substances, starch, oxidative degradation.

\section{INTRODUCCIÓN}

El desarrollo técnico y científico de los plásticos sintéticos potencializó su aplicación en la industria, convirtiéndose en el principal producto derivado de la petroquímica. Su demanda mundial ha aumentado junto con las economías emergentes, utilizando materias primas no renovables, tales como etileno, polipropileno (PP), copólimeros de vinilideno clorido (PVDC), polietileno tereftalato (PET), entre otros, que presentan, como efecto adverso, la acumulación de sus residuos sólidos, en razón de su baja capacidad de degradación (Iles \& Martin, 2013). En el 2014, la producción mundial de plásticos ascendió a $3,11 \times 10^{8}$ toneladas y para el mismo año, en Europa, el 39\% del plástico comercializado fue destinado para empaques (Selke et al. 2016). Los plásticos sintéticos tienen múltiples aplicaciones en diferentes áreas; tal es el caso de la industria de los alimentos, donde son utilizados como empaques para transportar, mantener o aumentar la vida útil de los mismos. Para el 2016, se produjeron en Colombia, $1,33 \times 10^{3}$ toneladas de resinas plásticas, donde el sector de empaques y envases representó el $56 \%$ en peso de las materias plásticas elaboradas (Acoplásticos, 2018).

El uso alternativo de compuestos renovables, como celulosa, almidón o quitina, en la elaboración de este tipo de empaques, difiere de los sintéticos, en términos de sostenibilidad, al mitigar el efecto invernadero, la emisión de gases, la acumulación de residuos sólidos y la polución, tras la cadena de producción y consumo, integrándose al ambiente como dióxido de carbono, agua y biomasa (De Vargas Mores et al. 2018). Por su parte, el uso de almidón de papa como materia prima en la elaboración de biopelículas es promisorio, al ser un compuesto abundante, de bajo costo y renovable, que confiere capacidad de degradación a las películas obtenidas. Dicho proceso requiere la adición de plastificantes, cuyo fin sea la reducción de las interacciones moleculares entre las cadenas de amilosa y amilopectina del almidón y, por tanto, su temperatura de transición vítrea (Luna et al. 2010). Uno de los principales inconvenientes en la elaboración de películas de almidón ha sido su elevada hidrofilicidad; de ahí, la necesidad de utilizar métodos que modifiquen la estructura química del polímero. Tal es el caso de la esterificación que, además, permite la optimización de sus propiedades mecánicas y el anclaje de moléculas orgánicas fotoactivas, cuyos rangos de absorción de luz logren disminuir la generación de procesos oxidativos en los alimentos (Cardenia et al. 2013).

Considerando que la producción de papa en Boyacá, para el 2016, fue de $6,7 \times 10^{5}$ toneladas (DANE, 2016), existe en el departamento una fuente de almidón residual derivado de la transformación de volúmenes importantes de papa a productos comerciales. La presente investigación tuvo como objetivo la obtención de biopelículas, a partir de la esterificación de este tipo de almidón y una mezcla de sorbitol- ácido acético como plastificante, incorporando en su formulación moléculas fotoactivas de origen natural: Rutina (Ruta graveolens-ruda), Betalaína (Beta vulgaris-remolacha) y Riboflavina comercial. Se evaluaron propiedades fisicoquímicas, mecánicas y de eficacia fotoactiva de las biopéliculas en carne de bovino (pierna).

\section{MATERIALES Y MÉTODOS}

Obtención del almidón. El almidón residual de papa (AN) fue obtenido a partir de la generación de residuos, durante la elaboración de productos fritos de papa, en la empresa Produfritos La Libertad (Duitama-Boyacá).

\section{Contenido de amilosa y amilopectina en almidón nativo} y modificado. La determinación de amilosa y amilopectina, se realizó con el método colorimétrico de Morrison \& Laignelet (1983), calculando el contenido de amilopectina por la diferencia entre el contenido de amilosa respecto al $100 \%$.

Modificación química del almidón: esterificación. Con el fin de anclar las sustancias orgánicas fotoactivas al almidón fue necesaria su esterificación, previa con anhídrido succínico, empleando piridina e hidróxido de sodio, como sistema catalizador (Martins et al. 2014). La esterificación del almidón nativo fue identificada mediante Espectroscopia de Infrarrojo Transformada de Fourier (por sus siglas en idioma inglés Fourier Transform Infrared Spectroscopy, FTIR) (Fang et al. 2004). La determinación del grado de sustitución (GS), se realizó de acuerdo con el método de titulación del succilinato, producido por la saponificación del almidón (Sodhi \& Singh, 2005).

Extracción de moléculas fotoactivas de origen natural. Las sustancias fotoactivas (rutina y betalaína) fueron obtenidas utilizando metanol como disolvente, mediante la extracción asistida por microondas. Para la identificación y la 
cuantificación de los extractos fue utilizada la espectroscopia UV-Vis, empleando los respectivos patrones comerciales.

Anclaje de las sustancias fotoactivas al almidón modificado. Una vez esterificado el almidón, se efectuó el anclaje de cada sustancia fotoactiva, mediado por un proceso de transesterificación, utilizando metanol como disolvente (Martins et al. 2014; Morrison \& Laignelet, 1983). Para los almidones modificados-fotoactivos y anclados a betalaína, rutina y riboflavina, se utilizaron las denominaciones AM-bet, AMRut y AM-Rib, respectivamente. Los tamaños de granulo de los almidones fueron analizados mediante microscopía electrónica de barrido (por sus siglas en idioma inglés Scanning Electronic Microscopy, SEM).

Elaboración de las biopelículas. Las biopelículas, se elaboraron por el método de gelatinización, suspendiendo cada uno de los almidones modificados-fotoactivos, en una mezcla de agua destilada y como plastificante sorbitol-ácido acético, con agitación y temperatura constante. La proporción almidón:plastificante fue de 4,0:2,5. Las suspensiones fueron vertidas en moldes antiadherentes de $20 \times 10 \mathrm{~cm}$ y secadas hasta la formación de cada biopelícula (Medina et al. 2012).

Evaluación fisicoquímica de las biopelículas. Teniendo en cuenta que las biopelículas tienen como finalidad su uso como empaque de alimentos, deben presentar baja solubilidad en agua y elevada transparencia. La solubilidad, se expresó como el porcentaje de materia seca, luego de $24 \mathrm{~h}$ de inmersión en agua; por su parte, la transmitancia fue determinada a una longitud de onda de $800 \mathrm{~nm}$, empleando un espectrofotómetro Thermo Scientific Genesys 10S Vis. La estabilidad frente a condiciones de $\mathrm{pH}$ extremos (2 y 12), se evaluó durante 30 días, por el seguimiento de los cambios en la apariencia física de los empaques, con valores entre 0 (inestable) y 10 (Muy estable) (Medina et al. 2012). Por último, el análisis térmico, se ejecutó mediante calorimetría diferencial de barrido y termogravimetría (DSC-TGA).

Evaluación mecánica de las biopelículas. Se evaluó la resistencia de tensión según la norma ASTM D638-10 (ASTM, 2003).

Evaluación de la eficacia fotoactiva de las biopelículas. La eficacia fotoactiva fue medida, a través del efecto en la disminución de la degradación oxidativa de material proteico y lipídico en carne de bovino. Experimentalmente, las muestras de carne fueron sometidas a tres condiciones: sin empaque, empaque de biopelícula de AN y empaques de biopelículas fotoactivas (AM-Bet, AM-Rut, AM-Rib). Cada una de estas condiciones fue analizada bajo dos sistemas de irradiación: Luz natural (117Watts $\left./ \mathrm{m}^{2}\right)$ e Irradiación UV (15,8 Watts $\left./ \mathrm{m}^{2}\right)$. Cada análisis fue llevado a cabo por triplicado, teniendo en cuenta, para cada caso, el respectivo control oscuro. La oxidación proteica, se determinó espectrofotométricamente, a $375 \mathrm{~nm}$, como resultado de la formación de complejos hidrazona (DNPH), entre los grupos carbonilo oxidados de residuos aminoacídicos y la 2,4-dinitrofenilhidrazina (DNP). La extracción y solubilización del material proteico requirió del empleo buffer fosfato $20 \mathrm{mM}$ pH 6,5 y ácido tricloroacético al $10 \%$. Previo a la lectura, los extractos fueron solubilizados en guanidina- $\mathrm{HCl} 6 \mathrm{M}$, bajo el buffer y $\mathrm{pH}$, empleado para la extracción (Oliver et al. 1987). Para la evaluación del daño oxidativo en lípidos, el extracto obtenido al emplear como solventes cloroformo:metanol (3:2), siguió el método yodométrico AOAC 965,33 (AOAC, 1990), que evalúa la presencia de peróxidos en grasas y en aceites, mediante la valoración del yodo, formado con tiosulfato de sodio.

\section{RESULTADOS Y DISCUSIÓN}

Contenido de amilosa y amilopectina en almidón nativo y modificado. El proceso de esterificación del almidón nativo solo afectó la composición química del componente amilosa. El porcentaje de amilosa para el almidón nativo fue $21,48 \pm 0,22$, mientras que para el almidón esterificado, $12,11 \pm 0,25$. Tal variación es debida a la esterificación de los grupos hidroxilo de la amilosa, que altera las interacciones propias de la estructura helicoidal del polímero con las moléculas de yodo (Sodhi \& Singh, 2005). En cuanto a los contenidos de amilopectina, tanto el almidón nativo como el almidón esterificado, presentaron contenidos del 78,52\%.

Espectroscopia infrarroja de almidones. En la figura 1, se muestra el espectro infrarrojo, obtenido para AN, AM, AMBet, AM-Rut y AM-Rib. AN presenta señales características a $3300 \mathrm{~cm}^{-1}$, correspondiente a las vibraciones del enlace de hidrógeno en los estiramientos libres (inter/intra-molecular) del grupo hidroxilo $(-\mathrm{OH})$, presentes en las unidades de anhidroglucosa de la amilosa y amilopectina (Ruiz-Avilés, 2006; Fang et al. 2004; Han et al. 2012). La señal débil en $2.930 \mathrm{~cm}^{-1}$ indica las vibraciones de extensión del enlace $\mathrm{C}-\mathrm{H}$, asociado al anillo de glucopiranosa; entre 923 y $1.162 \mathrm{~cm}^{-1}$, se observan las señales atribuidas al estiramiento del enlace $\mathrm{C}-\mathrm{O}$, características del almidón (Lopez-Rubio et al. 2009). La señal más importante que demuestra la efectividad de la esterificación de AN, se encontró cerca de $1.709 \mathrm{~cm}^{-1}$, asignada al estiramiento del grupo funcional carbonilo $(\mathrm{C}=\mathrm{O})$; de igual manera, la aparición de señales a $1.423,1.360$ y $1.220 \mathrm{~cm}^{-1}$ son asignadas a vibraciones de deformación antisimétrica de grupos $\mathrm{C}=\mathrm{O}$, vibración antisimetrica/simétrica de los grupos metilo $(-\mathrm{CH} 3)$ y vibraciones de estiramiento del grupo $\mathrm{C}=\mathrm{O}$, respectivamente (Heinze et al. 2006). Para los almidones esterificados y anclados, se observó un acortamiento en la banda, a $3.300 \mathrm{~cm}^{-1}$, indicando que los grupos $-\mathrm{OH}$ fueron reemplazados progresivamente por grupos funcionales éster 
menos hidrofílicos, que proporcionan impedimento estérico a las vibraciones por flexión (Rendón et al. 2011). En AMRut, se observó la aparición de cuatro bandas entre 1.600 y $1.450 \mathrm{~cm}^{-1}$, que dan cuenta de las vibraciones de los enlaces $\mathrm{C}=\mathrm{C}$, en sus anillos aromáticos, mientras que en $\mathrm{AM}-\mathrm{Bet} \mathrm{y}$ AM-Rib, se registraron señales cercanas a los $1.380 \mathrm{~cm}^{-1}$, relacionados con grupos fenólicos propios de las antocianinas (Juárez Montiel et al. 2013).
Determinación del grado de sustitución (GS). El GS en el almidón fue de 0,979, con un porcentaje de esterificación del $19 \%$, obtenido a partir de la saponificación de los grupos succinilato, formados en el proceso de modificación química. Este valor otorga al almidón modificado la potencial utilidad como empaque en la industria de alimentos, ya que los ésteres de almidón con grados de sustitución, entre 0,5 y 2,5 , tienen aplicaciones, como adhesivos, filtros de ciga-

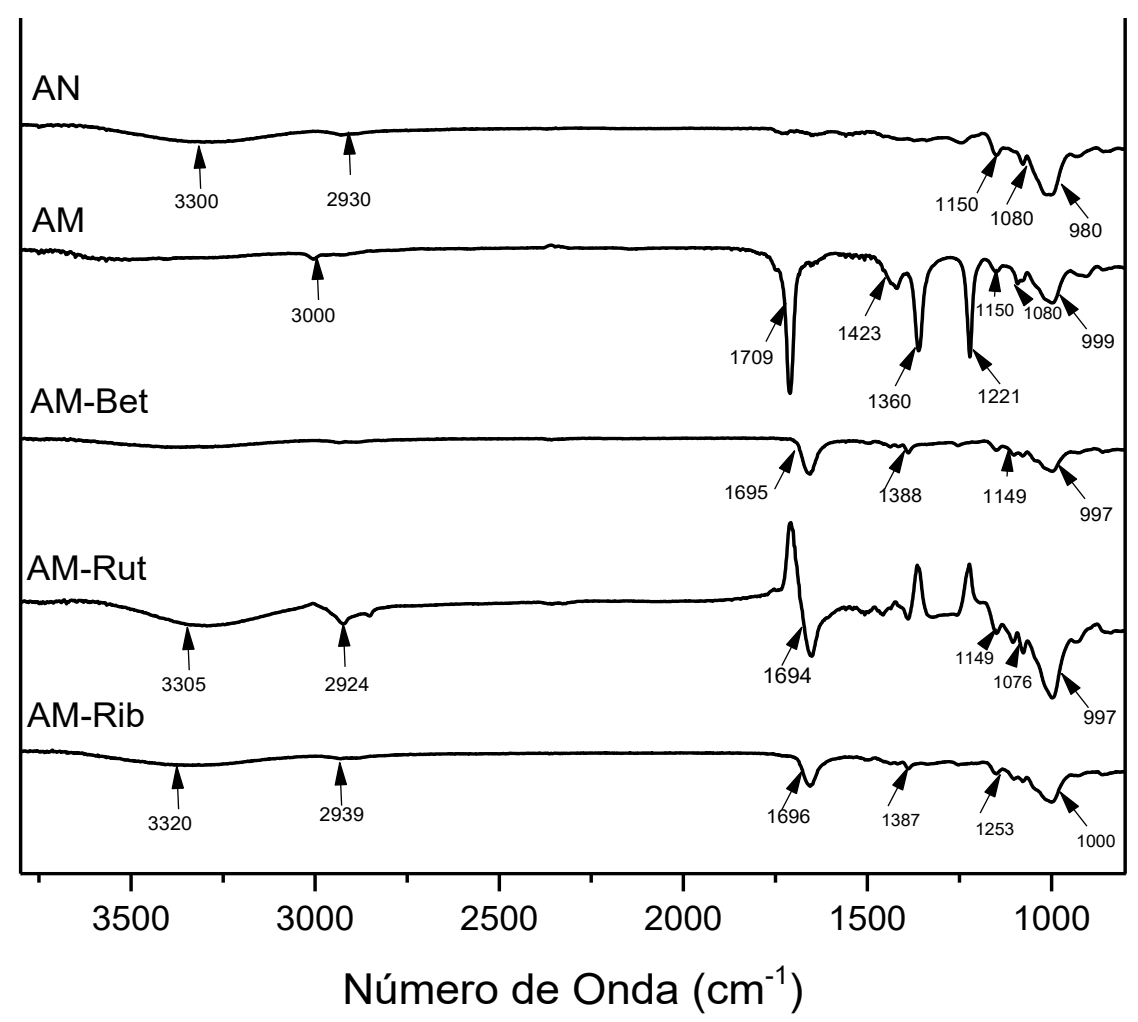

Figura 1. Espectro IR Almidón Nativo AN y modificados AM, AM-Bet, AM-Rut y AM-Rib.

rrillos, tabletas, plásticos biodegradables y adsorbentes de metales iónicos, en razón de su mayor hidrofobicidad. (Peñaranda et al. 2008).

Análisis térmogravimétrico de almidones. La tabla 1 muestra las temperaturas de gelatinización $(\mathrm{Tg})$ y de descomposición (Td), para AN, AM-Bet, AM-Rut y AM-Rib, parámetros que sirven como criterio de evaluación del cambio de estabilidad térmica del polímero, tras el proceso de esterificación. En el análisis térmico, se presentan dos reacciones endotérmicas: la primera, correspondiente a la gelatinización del almidón $\mathrm{Tg}$, con una disminución de peso, atribuida a la pérdida de agua. AN presentó una pérdida de $17,45 \%$ a una temperatura de $50,76^{\circ} \mathrm{C}$, valor comparable a los datos reportados en otros estudios (Ortega-Toro et al. 2015), mientras que en los almidones modificados, se obtuvieron
Tg menores: $47,13^{\circ} \mathrm{C}, 44,73^{\circ} \mathrm{C}$ y $45,89^{\circ} \mathrm{C}$, para AM-Rut, AM-Bet y AM-Rib, respectivamente, con pérdidas de peso de, aproximadamente, $13 \%$, como consecuencia de la desorganización de la estructura interna de los gránulos de almidón, específicamente, en las interacciones intra-intermoléculares del polímero esterificado. En la segunda reacción (descomposición térmica), se observó que los almidones modificados $\mathrm{y}$ anclados incrementaron su temperatura de descomposición (Td): $301,09^{\circ} \mathrm{C}, 311,4^{\circ} \mathrm{C}$ y $302,34^{\circ} \mathrm{C}$, para AM-Bet, AMRut y AM-Rib, respectivamente, comparado con la Td de AN $\left(299,85^{\circ} \mathrm{C}\right)$. Este parámetro, junto con la menor pérdida de peso (aproximadamente 10\%), indica que se obtuvieron polímeros de mayor estabilidad térmica (Mishra \& Rai, 2006; Schirmer et al. 2013). La evaluación de la entalpía $(\Delta H)$ es importante como indicador de la pérdida de orden molecular del gránulo (cristalinidad del almidón no gelatinizado). AN 
presentó una entalpía de gelatinización mayor a los almidones esterificados y anclados, resultado de una resistencia térmica, causada por componentes del gránulo, que se encuentran disueltos (amilosa, principalmente), lo que permite una capacidad de hinchamiento mayor, un tiempo de transición más largo y temperaturas pico más altas (Rendón et al. 2011).

Microscopia electrónica de barrido de almidones. Las morfologías de AN, AM-Rut, AM-Bet y AM-Rib, se muestran en la figura 2. Para el caso de AN (Figura 2a), se revelan variaciones de tamaño, con rangos entre $22-57 \mu \mathrm{m}$, evidenciando formas circulares, para los tamaños pequeños y ovaladas, para los tamaños grandes del rango. En general, se obtuvo un valor promedio de $32,53 \mu \mathrm{m}$, cercano al reportado en la literatura (Rendón et al. 2011). Al realizar los procesos de esterificación (introducción de grupos succínilo y anclaje de moléculas fotoactivas), se evidenció, tanto prevalescencia de las formas ovaladas como el aumento en tamaño del granulo:

Tabla 1. Análisis térmico DSC-TGA, para AN, AM-Bet, AM-Rut y AM-Bet.

\begin{tabular}{|c|c|c|c|c|c|c|}
\hline \multirow{2}{*}{ Biopelícula } & \multicolumn{3}{|c|}{ Primer pico endotérmico } & \multicolumn{3}{c|}{ Segundo pico endotérmico } \\
\cline { 2 - 7 } & $\mathrm{Tg}\left({ }^{\circ} \mathrm{C}\right)$ & $\boldsymbol{\Delta H}(\mathrm{J} / \mathrm{g})$ & $\% \mathrm{~W}(\mathrm{mg})$ & $\begin{array}{c}\mathrm{Td} \\
\left({ }^{\circ} \mathrm{C}\right)\end{array}$ & $\begin{array}{c}\Delta \mathrm{H} \\
(\mathrm{J} / \mathrm{g})\end{array}$ & $\% \mathrm{~W}(\mathrm{mg})$ \\
\hline AN & 50,76 & 688,7 & 17,45 & 299,85 & 333,5 & 80,00 \\
\hline AM-Bet & 44,73 & 682,3 & 13,96 & 301,09 & 90,99 & 70,65 \\
\hline AM-Rut & 47,13 & 647,1 & 13,25 & 311,4 & 153,4 & 72,99 \\
\hline AM-Rib & 45,89 & 615,2 & 13,16 & 302,34 & 108,7 & 71,94 \\
\hline
\end{tabular}

AM-Bet, 48,10 $\mu \mathrm{m}$; AM-Rut, 58,7 $\mu \mathrm{m}$ y AM-Rib, 58,64 $\mu \mathrm{m}$. Este incremento es debido a dos posibles efectos: 1) Generación de fuerzas de repulsión entre las moléculas de los AM, que aumentan los espacios inter/intra-moleculares y, 2) Coales- cencia de los gránulos, en razón de los puentes de hidrógeno presentes tras la introducción de grupos funcionales (succinílo y moléculas fotoactivas) (Rendón et al. 2011).

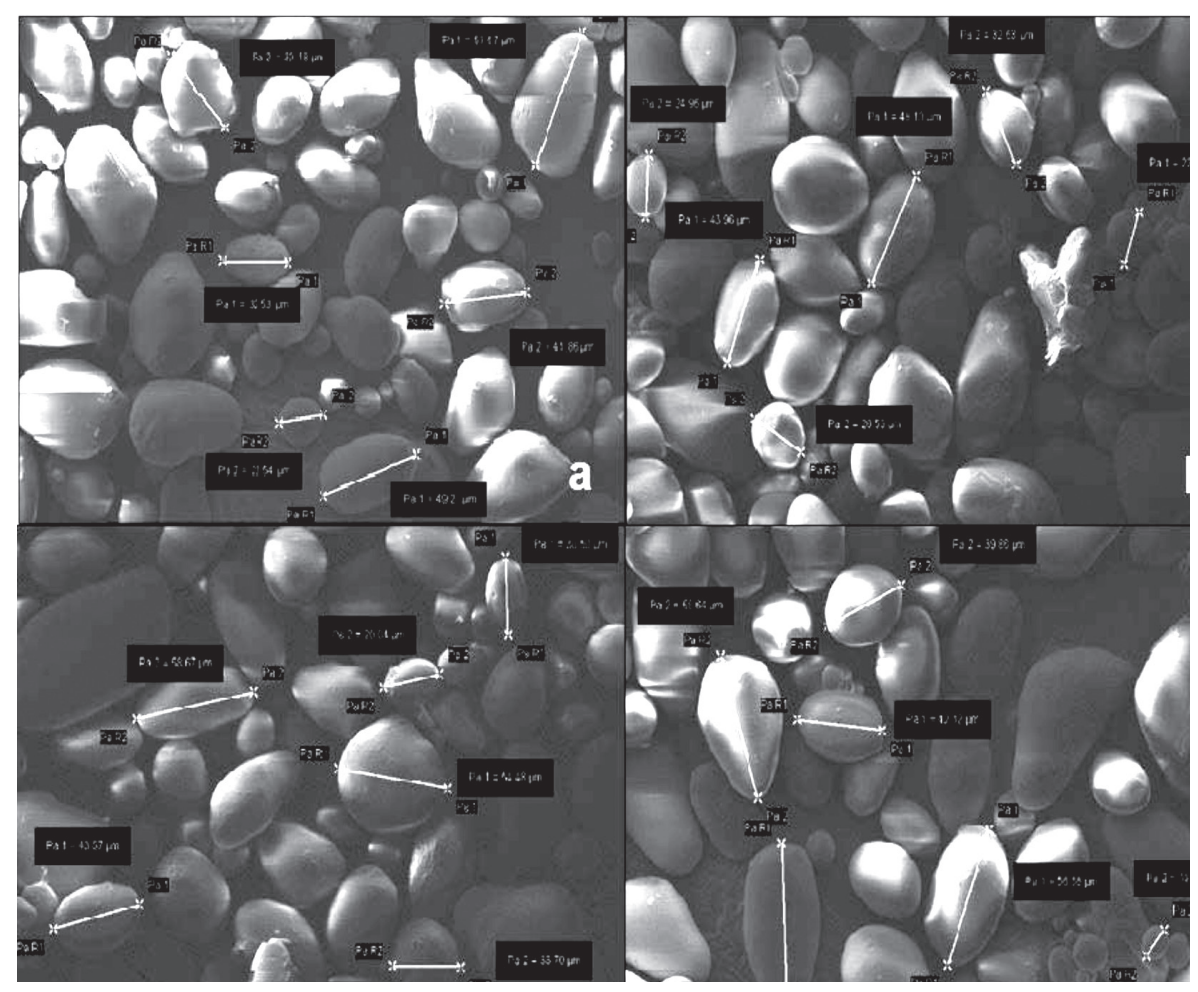

Figura 2. SEM 1000X. a) AN; b) AM-Bet; c) AM-Rut; d) AM-Rib. 
Evaluación fisicoquímica de las biopelículas. Transparencia: Los valores promedio para cada biopelícula indicaron que AM-Bet es la película más transparente: $84,9 \%$, debido a que su carácter hidrofilico favorece la retención de agua en su estructura y, por tanto, el paso de la luz, a $800 \mathrm{~nm}$. En contraste, una menor hidrofilicidad en AM-Rib y AM-Rut generó menores porcentajes de transparencia, $69,8 \%$ y $66,4 \%$, respectivamente; esta disminución es importante en empaques para alimentos, debido a que la penetración de la luz al empaque está involucrada con la ocurrencia de procesos oxidativos (Brody et al. 2001). Solubilidad: El porcentaje de solubilidad en agua disminuyó considerablemente, al emplear los almidones esterificados y anclados, ya que en el proceso se incorporan grupos funcionales menos hidrofílicos (Escobar, et al. 2009). Porcentajes de 24,3\%, para AM-Bet, 19,95\%; AM-Rut 19,95 y 10,27\%, AM-Rib, son menores a los reportados en otros estudios, indicando que la esterificación del almidón permite obtener materiales menos lábiles a condiciones húmedas y, por tanto, más óptimos para su uso industrial (Medina et al. 2012). Estabilidad en medio ácidobásico: En general, todas las biopelículas fotoactivas en condiciones ácidas ( $\mathrm{pH}$ 2) presentaron una alta estabilidad, con valores cercanos a 7, transcurridos 21 días de evaluación; sin embargo, durante el mismo periodo de tiempo y bajo condiciones básicas ( $\mathrm{pH} 12$ ), se observó una baja estabilidad, con valores próximos a 4; esto último, como consecuencia, ya sea de la hidrólisis de los grupos éster en medios muy básicos o por la formación de succilinatos con los iones $\mathrm{Na}^{+}$, aumentando, así, la hidrofilicidad del empaque (Mulhbacher et al. 2004).

Evaluación de las propiedades mecánicas de las biopelículas. Resistencia a la tensión. Los valores de tensión versus deformación son presentados en la tabla 2 . Se encontró que AM-Rib presentó la mayor tensión a la rotura $(6,83 \mathrm{MPa})$, lo cual, se podría justificar en que la molécula fotoactiva actúa como un agente de entrecruzamiento con el almidón, formando puentes de unión entre las cadenas del polímero, por tanto, mejorando su comportamiento mecánico (Ortega-Toro et al. 2015). De igual manera, fue posible observar que AM-Rut presentó propiedades mecánicas más bajas en comparación con las demás biopelículas $(6,241 \mathrm{MPa})$, afectando su estructura química y las propiedades mecánicas del polímero. La resistencia de tensión promedio de las biopelículas de almidón de papa modificado fueron mayores a los reportados en almidón de yuca, cal y fibras celulósicas, donde se encontraron valores de tensión promedio, de 1,7MPa (Wang et al. 2011). Durante la esterificación del almidón residual de papa con anhídrido succínico, se mejoraron las propiedades fisicoquímicas (hidrofilicidad, transparencia y estabilidad, en medios de $\mathrm{pH}$ extremos), mecánicas (resistencia a la ruptura) y térmicas (ahorros energéticos en el proceso de gelatinización), de las biopelículas obtenidas, proporcionándole así un potencial uso, como materia prima, en la elaboración de empaques biodegradables.

Evaluación de la eficacia de las biopelículas fotoactivas. La evaluación de la actividad de los empaques frente a condiciones de luz, se realizó por medio del efecto de los mismos, en la generación de daños oxidativos en proteínas y en lípidos de carne bovina (pierna), ya que dichos procesos están involucrados en la pérdida de calidad nutricional y sensorial de alimentos, así como el desarrollo de enfermedades crónicas y degenerativas en el consumidor (Cardenia et al. 2013). Cabe mencionar que, a partir de los rangos de absorción de cada una de las sustancias fotoactivas, se espera un efecto protector, así: AM-Bet, entre 400-600nm; AM-Rut, entre 190410nm y AM-Rib, entre 200-500nm, permitiendo analizar el efecto perjudicial de irradiación UV y luz natural. Oxidación proteica. La figura 3 expresa los resultados del daño oxidativo en nanomoles de DNPH por mg de proteína. La irradiación UV en ausencia de empaque provocó un daño oxidativo mayor a las proteínas presentes en alimentos cárnicos (3,0 nanomol DNPH/mg proteína), ya que esta irradiación es mucho más energética y potencialmente dañina, en comparación a la luz natural (0,5 nanomol DNPH/mg proteína, figura 3, sin empaque). Para las condiciones de irradiación UV, AM-Bet proporcionó mayor oxidación proteica $(2,1$ nanomol $\mathrm{DNPH} / \mathrm{mg}$ proteína), comparado con AM-Rib (1,75 nanomol $\mathrm{DNPH} / \mathrm{mg}$ proteína) y AM-Rut (1,0 nanomol DNPH/mg proteína); bajo irradiación UV, AM-Rut disminuyó la degradación proteica en un $66,6 \%$, comparado con la muestra sin empaque. En cuanto a la irradiación con luz natural, las biopelículas de AM-Rut (0,24 nanomol DNPH/mg proteína) indicaron una disminución del daño oxidativo del $50,4 \%$, presentando un efecto protector superior frente a los demás tipos de ensayos; sin embargo, al realizar el análisis ANOVA entre las biopelículas fotoactivas, no se encontraron diferencias signi-

Tabla 2. Propiedades mecánicas de las biopelículas.

\begin{tabular}{|c|c|c|}
\hline Biopelícula & Resistencia a la tensión $\mathbf{( M P a )}$ & Deformación máxima $(\mathbf{m m})$ \\
\hline AM-Bet & 6,45 & 15,95 \\
\hline AM-Rut & 6,24 & 28,95 \\
\hline AM-Rib & 6,83 & 6,13 \\
\hline
\end{tabular}


ficativas $(p>0,05)$. El control oscuro presentó valores menores, a 0,01 nanomol DNPH/mg proteína, haciendo evidente que el daño oxidativo proteico es generado exclusivamente por la acción de la luz. A partir de los resultados expresados en la figura 3, se puede afirmar que, biopelículas fotoactivas, con rango de absorción cercano a longitudes de onda entre 200-400nm, generan un efecto protector de los alimentos, manteniendo la estructura química de las proteínas y, por tanto, su calidad organoléptica y nutricional. Oxidación lipídica: La oxidación lipídica en alimentos cárnicos depende de la producción de compuestos de bajo peso molecular (cetonas, aldehídos y alquenos); además, es la segunda causa de deterioro, después del efecto microbiano. Dentro de las principales alteraciones físicas por este tipo de oxidación, se encuentran cambios en el aroma, sabor (enranciamiento) y color (producción de metamioglobina- metahemoglobina), además de la pérdida de nutrientes y la formación de sustancias potencialmente nocivas, como es el caso de los óxidos de colesterol y especies radicalarias (Cardenia et al. 2013). Los resultados obtenidos son presentados en la figura 4 y expresados como miliequivalentes de $\mathrm{O}_{2}$ activo por $\mathrm{kg}$ de muestra (AOAC, 1990). Para condiciones de luz natural, la biopelícula que indicó una mayor efectividad fue AM-Bet (7,23 Meq O2/kg muestra), con una disminución del 67,14\%, en la producción de peróxidos, frente a la muestra sin empaque (22 Meq $\mathrm{O}_{2} / \mathrm{kg}$ muestra); lo anterior es atribuido a que, aunque su transparencia es mayor, su capacidad de absor- ción, entre los 400-600nm, impide la interacción luz-muestra, en la atmósfera interior del empaque. Bajo condiciones de irradiación UV, la biopelícula que presentó una mayor eficiencia fue AM-Rut (5,98 Meq $\mathrm{O}_{2} / \mathrm{kg}$ muestra), disminuyendo en un $57,82 \%$ la producción de peróxidos, en comparación con la muestra sin empacar (14 Meq $\mathrm{O}_{2} / \mathrm{kg}$ muestra), en razón a que la molécula rutina absorbe energía en las regiones UV-B y UV-C, que son potencialmente energéticas y capaces de producir alteraciones físicas, en ácidos grasos insaturados, colesterol y proteínas (Ladeira et al. 2014). El control oscuro presentó niveles despreciables de peróxidos en las muestras ( $<0,05 \mathrm{Meq} \mathrm{O}_{2} / \mathrm{kg}$ muestra).

El análisis de varianza ANOVA demostró que las biopelículas fotoactivas tienen diferencias significativas en la reducción del índice peróxidos de las muestras de carne bovina $(p<0,05)$, expuestas a condiciones, tanto de irradiación UV como luz natural. La prueba de comparaciones múltiples (Tukey) determinó que la eficacia fotoactiva del bioplástico, elaborado con Almidón Nativo AN, es significativamente menor, comparada con los bioplásticos fotoactivos de AM-Bet, AM-Rut y AM-Rib, lo que implica que el anclaje de moléculas fotoactivas inhibe la generación de reacciones oxidativas lipídicas en cadena.

De esta investigación se concluye que la modificación química del almidón, para su posterior unión a moléculas fotoac-

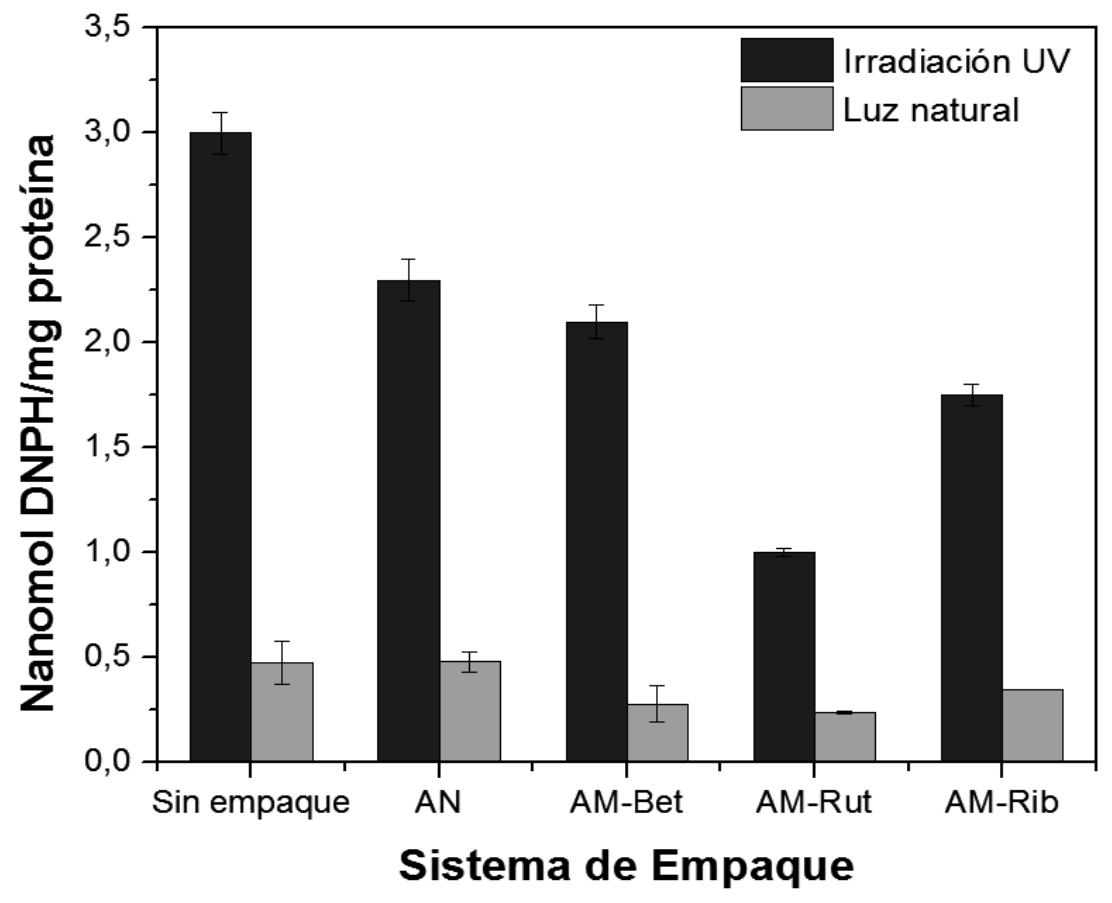

Figura 3. Daño oxidativo en proteínas de carne de bovino a diferentes condiciones de empacado e irradiación. 


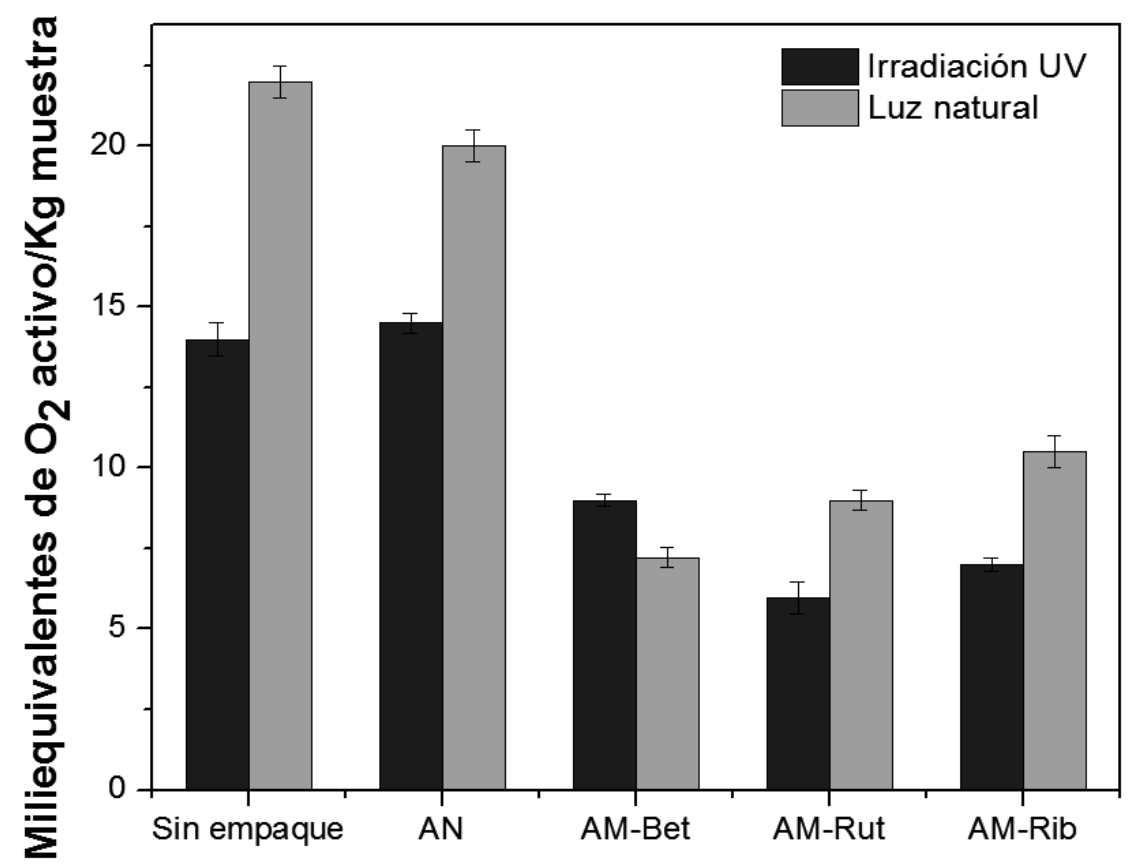

Sistema de Empaque

Figura 4. Daño oxidativo en lípidos de muestras a diferentes condiciones de empacado e irradiación.

tivas, representa una alternativa en la industria de empaques alimentarios, dada su capacidad de protección de productos, que sean sensibles a la acción de la luz. El empleo de biopelículas fotoactivas, además de presentar mejoras en las propiedades fisicoquímicas y mecánicas con respecto a otro tipo de empaques biodegradables, demostró la reducción de procesos oxidativos y radicalarios al interior del empaque. Particularmente, la biopelícula de AM-Rut evidenció los mejores resultados en la reducción de procesos oxidativos proteicos y lipídicos.

Recomendaciones: Se sugiere el empleo de diferentes moléculas fotoactivas, que amplíen los rangos de absorción empleados, principalmente, en los rangos visible-infrarrojo. A su vez, se propone el estudio de diferentes variables, tales como concentración de oxígeno, temperatura, cantidad y tipo de radiación, que afecten la ocurrencia de procesos oxidativos en alimentos fácilmente perecederos.

Agradecimientos: Los autores expresan su agradecimiento al Instituto para la Investigación e Innovación en Ciencia y Tecnología de Materiales "INCITEMA", por su colaboración en los análisis de pruebas de tensión. Lina Johanna Niño agradece a la convocatoria 775 de Colciencias, por el otorgamiento de la beca-pasantía de jóvenes investigadores por la paz. Carlos Iván Rojas agradece a la convocatoria 733 de Colciencias, por el otorgamiento de la beca para la forma- ción de capital humano de alto nivel en el departamento de Boyacá. Conflictos de intereses: El manuscrito fue preparado y revisado con la participación de todos los autores, quienes declaramos que no existe conflicto de intereses que ponga en riesgo la validez de los resultados presentados. Financiación: Este estudio fue financiado por la Dirección de Investigaciones DIN-UPTC, al proyecto titulado "Elaboración de Bioplásticos Fotoactivos y su Aplicación en el Empaque de Alimentos Sensibles a la Oxidación", SGI 1921.

\section{REFERENCIAS}

1. ACOPLÁSTICOS. 2018. Plásticos en Colombia. Plásticos, química, petroquímica, cauchos, pintura, tintas, fibras. p. 208-210. Disponible desde Internet en: https://www.acoplasticos.org/index.php/mnunoti/143-ns-170907 (con acceso 23/02/2018).

2. AOAC. 1990. Official Methods of Analysis. Association of Official Analytical Chemists (EUA). 15th (1):136-138.

3. ASTM. 2003. Standard Test Method for Tensile Properties of Plastics, p.8.

4. BRODY, A.; STRUPINSKY, B.; KLINE, L. 2001. Active packaging for food applications. Ed. Technomic Publishing Co (Lancaster). p.236-340. 
5. CARDENIA, V.; RODRIGUEZ-ESTRADA, M.T.; BOSELLI, E.; LERCKER, G. 2013. Cholesterol photosensitized oxidation in food and biological systems. Biochimie. 95(3):473-481. https://doi.org/10.1016/j. biochi.2012.07.012

6. DEPARTAMENTO ADMINISTRATIVO NACIONAL DE ESTADÍSTICA -DANE-. 2016. Encuesta anual manufacturera. Disponible desde Internet en: https://www. dane.gov.co/files/investigaciones/boletines/eam/ boletín_eam_2016.pdf (con acceso 23/02/2018).

7. DE VARGAS MORES, G.; PAULETTO, C.; FINOCCHIO, S.; BARICHELLO, R.; AVILA, E. 2018. Sustainability and innovation in the brazilian supply chain of green plastic. J. Cleaner Production. 177:12-18. https:// doi.org/10.1016/j.jclepro.2017.12.138

8. ESCOBAR, D.; SALA, A.; SILVERA, C.; HARISPE, R.; MÁRQUEZ, R. 2009. Películas biodegradables y comestibles desarrolladas en base a aislado de proteínas de suero lácteo: estudio de dos métodos de elaboración y del uso de sorbato de potasio como conservador. INNOTEC. 4:33-36.

9. FANG, J.M.; FOWLER, P.A.; SAYERS, C.; WILLIAMS, P.A. 2004. The chemical modification of a range of starches under aqueous reaction conditions. Carbohydrate Polymers. 55(3):283-289. https://doi. org/10.1016/j.carbpol.2003.10.003

10. HAN, F.; LIU, M.; GONG, H.; LÜ, S.; NI, B.; ZHANG, B. 2012. Synthesis, characterization and functional properties of low substituted acetylated corn starch. Internal J. Biological Macromolecules. 50(4):1026-1034. https://doi.org/10.1016/j.ijbiomac.2012.02.030

11. HEINZE, T.; LIEBERT, T.; KOSCHELLA, A. 2006. Esterification of polysaccharides. Springer-Berlin Verlag Heidelberg (New York). p.143-147.

12. IIES, A.; MARTIN, A. 2013. Expanding bioplastics production: Sustainable business innovation in the chemical industry. J. Cleaner Production. 45:38-49. https://doi.org/10.1016/j.jclepro.2012.05.008

13. JUÁREZ MONTIEL, R.G.; PINTO APAZA, N.; CANO DE TERRONES, T. 2013. Polimerización de compuestos antociánicos presentes en el colorante de la hierba santa (Cestrum hediondinum D.) y prueba de adsorción de iones aluminio (III). Rev. Soc. Quím. Perú. 79(1):71-79.
14. LADEIRA, M.M.; SANTAROSA, L.C.; CHIZZOTTI, M.L.; RAMOS, E.M.; MACHADO NETO, O.R.; OLIVEIRA, D.M.; RIBEIRO, J.S. 2014. Fatty acid profile, color and lipid oxidation of meat from young bulls fed ground soybean or rumen protected fat with or without monensin. Meat Sci. 96(1):597-605. https:// doi.org/10.1016/j.meatsci.2013.04.062

15. LOPEZ-RUBIO, A.; CLARKE, J.M.; SCHERER, B.; TOPPING, D.L.; GILBERT, E.P. 2009. Structural modifications of granular starch upon acylation with short-chain fatty acids. Food Hydrocolloids. 23(7):1940-1946. https://doi.org/10.1016/j. foodhyd.2009.01.003

16. LUNA, G.; VILLADA, H.; VELASCO, R. 2010. Almidón termoplástico de yuca reforzado con fibra de fique: Preliminares. Dyna. 76(159):145-51.

17. MARTINS, L.; LOBO, B.; VINÍCIUS, L.; FRANCISCO DE AQUINO, S.; FRÉDÉRIC, L. 2014. Application of cellulose-immobilized riboflavin as a redox mediator for anaerobic degradation of a model azo dye remazol golden yellow RNL. Industrial Crops and Products. 65:454-462. https://doi.org/10.1016/j. indcrop.2014.10.059

18. MEDINA, O.J.; PARDO, O.H.; ORTIZ, C.A. 2012. Modified arracacha starch films characterization and its potential utilization as food packaging. Vitae. (Colombia). p.186-196.

19. MISHRA, S.; RAI, T. 2006. Morphology and functional properties of corn, potato and tapioca starches. Food Hydrocolloids. 20:557-566. https://doi. org/10.1016/j.foodhyd.2005.01.001

20. MORRISON, W.R.; LAIGNELET, B. 1983. An Improved colorimetric procedure for determining apparent and total amylose in cereal and other starches. J. Cereal Science. 1(1):9-20. https://doi.org/10.1016/S073 3-5210(83)80004-6

21. MULHBACHER, J.; ISPAS-SZABO, P.; MATEESCU, M.A. 2004. Cross-linked high amylose starch derivatives for drug release: Il. Swelling properties and mechanistic study. International J. Pharmaceutics. 278(2):231238. https://doi.org/10.1016/j.ijpharm.2004.03.008

22. OLIVER, C.N.; AHN, B.W.; MOERMANS, E.J.; GOLDSTEIN, S.; STADTMAN, R. 1987. Age-related changes in oxidized proteins. J. Biological Chemistry. 262(12):5488-5491. 
23. ORTEGA-TORO, R.; TALENS, P.; CHIRALT, A. 2015. Influence of citric acid on the properties and stability of starch-polycaprolactone based films. J. Applied Polymer Science. 132(2):1-16. https://doi. org/10.1002/app.42220

24. PEÑARANDA, O.I.; PERILLA, J.E.; ALGECIRA, N.A. 2008. Revisión de la modificación química del almidón con ácidos orgánicos. Ingeniería e Investigación. (Colombia). 28(3):47-52.

25. RENDÓN, R.; GARCÍA, E.; GUIZADO, M.; SALGADO R.; RENGEL, N. 2011. Obtención y caracterización de almidón de platano (Musa paradisiaca L.) acetilado a diferentes grados de sustitución. Afinidad LXV. p.294-300.

26. RUIZ AVILÉS, G. 2006. Obtención y caracterización de un polímero biodegradable a partir del almidón de yuca. Ingeniería y Ciencia. (Colombia). 2(4):5-28.

27. SCHIRMER, M.; HÖCHSTÖTTER, A.; JEKLE, M.; ARENDT, E.; BECKER, T. 2013. Food hydrocolloids physicochemical and morphological characterization of different starches with variable amylose/ amylopectin ratio. Food Hydrocolloids. 32(1):52-63. https://doi.org/10.1021/jf904531d
28. SELKE, S.E.M.; CULTER, J.D.; HERNANDEZ, R.J. 2016. Plastics packaging: properties, processing, Applications and Regulations. Carl Hanser Verlag, 2nd Edition (Munich). 448p.

29. SODHI, N.S.; SINGH, N. 2005. Characteristics of acetylated starches prepared using starches separated from different rice cultivars. J. Food Engineering. 70(1):117-127. https://doi. org/10.1016/j.jfoodeng.2004.09.018

30. WANG, S.; SHARP, P.; COPELAND, L. 2011. Structural and functional properties of starches from field peas. Food chemistry. 126(4):1546-1552. https://doi. org/10.1016/j.foodchem.2010.11.154

Recibido: Febrero19 de 2018

Aceptado: Noviembre 13 de 2018

\section{Cómo citar:}

Niño Otálora, L.J.; García Torres, A.M.; Medina Vargas, O.J.; Rojas Morales, C.I. 2018. Biopelículas fotoactivas: material de empaque en alimentos sensibles a la oxidación. Rev. U.D.C.A Act. \& Div. Cient. 21(2):457-466. https://doi.org/10.31910/ rudca.v21.n2.2018.1080 